\title{
Systems Engineering Challenges for GSFC Space Science Mission Operations
}

\author{
Julie Thienel \\ NASA Goddard Space Flight Center \\ 8800 Greenbelt Road \\ Greenbelt, MD 20771 \\ 310-614-7016 \\ julie.thienel@nasa.gov
}

\begin{abstract}
The NASA Goddard Space Flight Center Space Science Mission Operations (SSMO) project currently manages 19 missions for the NASA Science Mission Directorate, within the Planetary, Astrophysics, and Heliophysics Divisions. The mission lifespans range from just a few months to more than 20 years. The WIND spacecraft, the oldest SSMO mission, was launched in 1994. SSMO spacecraft reside in low earth, geosynchronous, highly elliptical, libration point, lunar, heliocentric, and Martian orbits. SSMO spacecraft range in size from 125 kg (Aeronomy of Ice in the Mesosphere (AIM)) to over 4000 kg (Fermi Gamma-Ray Space Telescope (Fermi)). The attitude modes include both spin and three-axis stabilized, with varying requirements on pointing accuracy. The spacecraft are operated from control centers at Goddard and off-site control centers; the Lunar Reconnaissance Orbiter (LRO), the Solar Dynamics Observatory (SDO) and Magnetospheric MultiScale (MMS) mission were built at Goddard. The Advanced Composition Explorer (ACE) and Wind are operated out of a multi-mission operations center, which will also host several SSMO-managed cubesats in 2017. This paper focuses on the systems engineering challenges for such a large and varied fleet of spacecraft.
\end{abstract}

\section{Table of Contents}

1. INTRODUCTION...............................

2. SUBSYSTEM ANOMALY OVERVIEW ..............2

3. MISSION OPERATIONS IMPROVEMENTS .......... 5

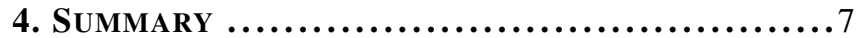

APPENDICES.................................... 7

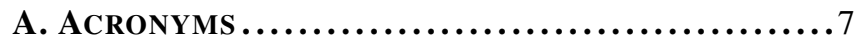

ACKNOWLEDGMENTS ............................ 7

REFERENCES .................................. 7

BIOGRAPHY $\ldots \ldots \ldots \ldots \ldots \ldots \ldots \ldots \ldots \ldots \ldots \ldots \ldots \ldots, \ldots$

\section{INTRODUCTION}

The NASA Goddard Space Flight Center (GSFC), named for rocket pioneer Dr. Robert H. Goddard, was established in May 1,1959. GSFC builds spacecraft and instruments, and develops new technology to study earth, the sun, the solar system, and the universe. The prime focus areas are earth science, astrophysics, heliophysics, planetary science, space communications and navigation, and suborbital platforms and ranging services [1]. The Space Science Mission Operations (SSMO) project manages astrophysics, heliophysics, and planetary science missions for the NASA Science Mission Directorate. Table 1 lists the SSMO-managed missions, their launch dates and orbital regimes. (See Appendix A for acronym definitions.)

U.S. Government work not protected by U.S. copyright

\author{
Richard R. Harman \\ NASA Goddard Space Flight Center \\ 8800 Greenbelt Road \\ Greenbelt, MD 20771 \\ 301-286-9613 \\ richard.r.harman@nasa.gov
}

Table 1. SSMO Missions

\begin{tabular}{|c|c|c|}
\hline Mission & Launch Date & Orbit Regime \\
\hline \hline ACE & 1997 & L1 \\
AIM & 2007 & LEO \\
ARTEMIS & 2007 & Lunar/Lunar L1 \\
Fermi & 2008 & LEO \\
IBEX & 2008 & HEO \\
IRIS & 2013 & LEO \\
LRO & 2009 & Lunar \\
MAVEN & 2013 & Mars \\
MMS & 2015 & HEO \\
OSIRIS-REx & 2016 & Asteroid \\
RHESSI & 2002 & LEO \\
SDO & 2010 & GEO \\
SOHO & 1995 & L1 \\
STEREO & 2006 & Heliocentric \\
Swift & 2004 & LEO \\
THEMIS & 2007 & HEO \\
TIMED & 2001 & LEO \\
WIND & 1994 & L1 \\
Van Allen Probes & 2012 & HEO \\
\hline
\end{tabular}

This paper focuses on the system engineering challenges for such a large and varied fleet of spacecraft. Attitude control, propulsion, and power subsystems will be discussed. LRO, MMS, IRIS, Fermi and IBEX all have had star tracker resets, due to radiation hits, poor star fields, occultation, and increasing hot spots. SDO, STEREO, and Solar and Heliophysics Observatory (SOHO) have had gyro issues such as elevated currents and failures, which ultimately led to the loss of STEREO B and a "Mission Interruption" for SOHO [2]. Some of the older spacecraft have battery management and solar array issues. Both LRO and Fermi face reaction wheel degradation concerns. ACE faced concerns about the amount of fuel remaining when maneuvers started to underperform at the same time as tank temperature readings began to increase. In addition to the effort required to maintain the orbits of these spacecraft, close approaches with space debris and impact with micrometeoroids also present challenges. MMS includes four identical formation flying spacecraft in highly elliptical orbits and has likely been impacted by an unidentified object.

In addition to investigations of anomalies, improvements in mission operations are an ongoing effort. A tool is in devel- 
opment for LRO that accurately predicts angular momentum buildup in order to lengthen the time between momentum unloads and thereby maximize science return. Gyro-less operation modes are in development for both LRO and SDO. Improved tools have been created for monitoring trends in reaction wheel drag torque, star tracker residuals, and gyro biases. Supporting such a wide variety of missions brings numerous challenges but also numerous rewards in witnessing the scientific discoveries that have resulted.

\section{Subsystem Anomaly OVerview}

The SSMO missions have experienced a wide range of anomalies both inflight and in the supporting ground systems. The focus here will be on select inflight anomalies classified by spacecraft subsystem.

\section{Attitude Control}

Gyros-Both STEREO and SOHO have experienced gyro failures. The SOHO gyros failed after recovery from the 1998 inflight mishap[2]. SOHO, a joint ESA/NASA observatory, has been on orbit for 21 years. SOHO studies the internal structure of the sun, producing images critical to space weather monitoring and prediction. The gyros were not required for science operations; the gyros were used in the safe mode and in momentum management. Operations have continued through gyro-less momentum management, so attitude control can continue with reaction wheels rather than thrusters, saving fuel and resulting in an additional 18 years of solar science.

STEREO, launched in 2006, suffered three gyro failures, all in the X-axis gyro direction. STEREO provided the first ever stereoscopic measurement to study the sun and space weather. The IMU-1 failed on STEREO Ahead shortly after launch in 2007. IMU-1 in STEREO Behind was nearing the end of life in late 2012 and was powered off. In early 2014, IMU-2 failed on STEREO Behind. Both spacecraft began reduced gyro operations to preserve life. The gyros remain powered off except for momentum unloads, special observations, or if the star tracker fails to acquire after power on. Both spacecraft entered solar conjunction in 2015. In preparation for solar conjunction, the operations team exercised the hardware command loss timers (CLT) on each spacecraft. The CLT resets the spacecraft processor if no commands are received within a 72 hour time period. Conjunction operations were successfully tested on Ahead in July 2014. In September 2014 the same testing was performed on Behind and at the completion of the test on October 1, 2014 communication was lost on Behind. The cause was a failure of the IMU-1 $\mathrm{X}$-axis gyro. Following the CLT reset the star tracker failed to acquire within 60 seconds, resulting in powering on of IMU-1. The X-axis failed, resulting in a large (false) angular momentum followed by an attempt to dump momentum for up to 7 minutes. The end state of the spacecraft was likely a tumble that evolved into a spin about the principal inertia axis, with little to no sun on the solar arrays, draining the battery and ultimately resulting in frozen components including the hydrazine propellant.

STEREO Ahead exited conjunction in July 2015 and continues to operate in a reduced gyro mode. Contact was made with STEREO Behind on August 21, 2016. Efforts at recovery continued aggressively through late September when the spacecraft began to drift out of range for communication with the deep space network. Prior to the loss of communication several attempts were made to slowly recharge the battery and thaw the hydrazine. The efforts were difficult due to the very low data rate and limited ability to receive onboard telemetry. Doppler analysis suggested that at the time of the first contact the system momentum was likely on the order of $30 \mathrm{Nms}$. After the propulsion tanks and at least one system of propellant lines thawed, at least two attempts to unload system momentum likely occurred. The system momentum dropped to approximately $22 \mathrm{Nms}$, but then another anomaly occurred resulting in an increased spin rate and estimated momentum of $60 \mathrm{Nms}$. Commands will continue to be sent to the spacecraft in an attempt to carefully manage the battery charging as the spacecraft solar arrays become intermittently illuminated. The STEREO operations team put in an incredible effort to recover STEREO Behind and are well prepared to continue the effort as the orbital geometry improves again in several months.

Star Trackers-Several SSMO missions have also experienced star tracker anomalies. IBEX entered safe mode in February 2016 due to erroneous data from the star tracker. IBEX, a heliophysics mission, studies the interaction between the solar wind and the interstellar medium at the edge of our solar system. IBEX spins at $4 \mathrm{rpm}$ and is in a highly elliptic orbit $(1.1 \mathrm{Re} \times 50 \mathrm{Re})$. The problem was isolated to an FPGA cell that may have suffered a micro-latch-up in the $4 \mathrm{~Hz}$ processing utilized by IBEX. The FPGA cannot be reconfigured on the older ASC on IBEX, so a work around was developed that merged the $8 \mathrm{~Hz}$ and $2 \mathrm{~Hz}$ modes. The updates have been uploaded to the spacecraft, allowing operations to return to normal.

IRIS, also a heliophysics mission launched in 2013, observes how solar material moves, gathers energy, and heats up as it travels through the sun's lower atmosphere. IRIS aborted a roll maneuver in October 2015 and entered its safe coarse control mode after both star tracker heads were unable to produce a valid attitude. Previous anomalies occurred in 2013 and 2015, all related to prolonged star tracker outages. The errors were traced to three factors. First, the ACS does not correctly account for the star tracker time tag, so when the spacecraft is rolling for science observations there is a delay in the attitude solution used by the ACS. Second, the outages occurred during meager star fields, further degrading the attitude solution. Finally, the star tracker CCD experienced a medium to high dose of radiation during the end of the solar cycle. The star tracker is now operating in a high rate mode, which improves the time tag issue associated with the centroiding of the estimate. IRIS has returned to its rolling mode for science observations. The star tracker performance is degraded but is within the IRIS pointing specifications.

The Fermi Gamma Ray Telescope, launched in 2008, observes the cosmos using the highest-energy form of light. Mapping the entire sky every three hours, Fermi provides an important window into the most extreme phenomena of the universe, from gamma-ray bursts and black-hole jets to pulsars, supernova remnants and the origin of cosmic rays. In September 2016 with data from Fermi and other facilities, an international team of scientists found the first gamma-ray binary in another galaxy and the most luminous one ever seen. The dual-star system, dubbed LMC P3, contains a massive star and a crushed stellar core that interact to produce a cyclic flood of gamma rays. [15] In 2012 Fermi experienced invalid solutions from the two operational star trackers for extended periods of time due to increased hot spots. CCD image dumps revealed a cluster of warm pixels as well as other warm pixels on the CCD. The cluster of warm pixels would cause the tracker to lose lock on stars in the FOV, resulting in an invalid 


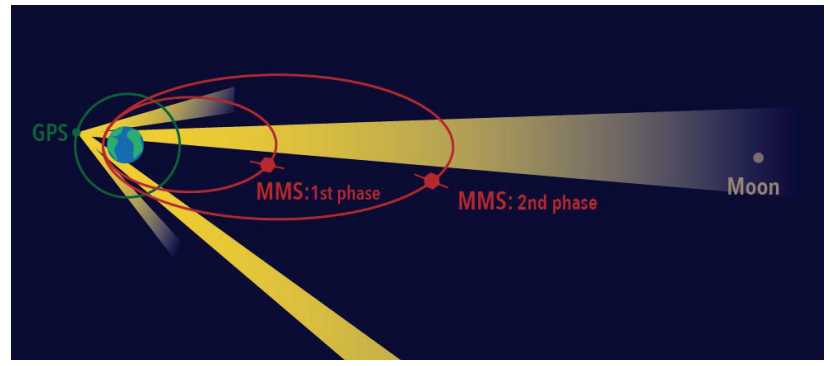

Figure 1. MMS and GPS Orbits

attitude solution. Both star tracker thermal electric cooler set points were lowered from 0 deg $C$ to $-10 \mathrm{deg} C$ which reduced the warm pixel locations. Fermi now downloads CCD images quarterly to monitor for warm pixels.

The MMS mission, launched in 2015, comprises four identical spinning spacecraft flying in highly elliptical orbits. The spacecraft form a tetrahedron at apogee, sampling the boundary of the magnetopause and the plasma processes in an attempt to gain understanding of the process of magnetic reconnection. MMS has achieved several significant milestones. First, the MMS Navigator, responsible for the onboard orbit estimation, set a record for the highest-ever reception of signals and onboard navigation solutions by an operational GPS receiver in space. At perigee, Navigator set a record as the fastest operational GPS receiver in space. Figure 1 depicts the MMS orbits with the GPS orbit. [3][4] In September 2016 the four MMS spacecraft formed a 7-km tetrahedron, the closest separation ever of a multi-spacecraft formation. In early 2017 MMS will undergo 32 long maneuvers to raise apogee to 25 earth radii, another test for the Navigator.

Early in the mission MMS experienced a number of reboots of their star trackers. Each spacecraft is equipped with four camera heads and a single processing unit. Early in the mission, following the deployment of the axial booms, it was determined that reflections from one boom blinded the field of view of one camera head. That head was removed from the onboard attitude estimation. The spacecraft spin at 3.05 $\mathrm{rpm}$ and the trackers operate in a high rate centroiding mode. The reboots have mostly been caused by high energy particleinduced single event upsets to the FPGA. Two software updates have been developed to improve the performance of the star trackers. The first updated the bright object centroiding function to reduce the number of invalid measurements caused by boom reflections. The second update introduced instrument failure detection which first reloads the FPGA to quickly restore performance of the star tracker following an SEU, and if that fails it causes an autonomous reboot. Since the two software updates three prolonged outages have occurred. One prolonged outage was the result of a possible micrometeoroid hit on MMS4. Most resets now are false positives resulting from earth occultation near perigee, causing the new failure detection to trip. Recently the star trackers appeared to be picking up bright objects near apogee. A check of the line of sight between spacecraft indicates that the star trackers are imaging some of the other spacecraft in the tighter $7 \mathrm{~km}$ formation (Figure 2).

LRO, launched in 2009, contains seven instruments and has made numerous groundbreaking discoveries of the moon. In 2015, the LRO Camera (LROC) captured a stunning view of Earth from the moon. (Figure 3) Over the mission lifetime,

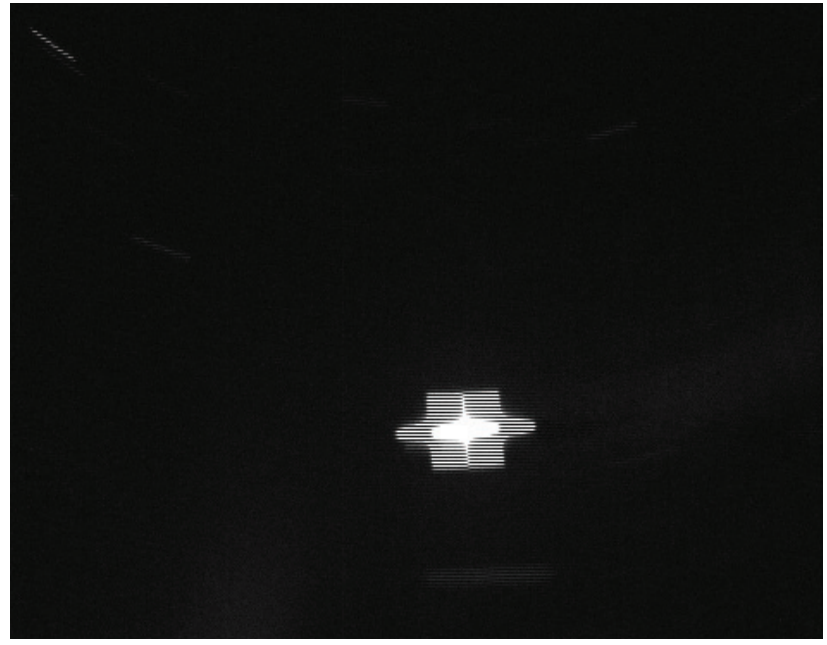

Figure 2. MMS1 as Observed by MMS4 Star Tracker

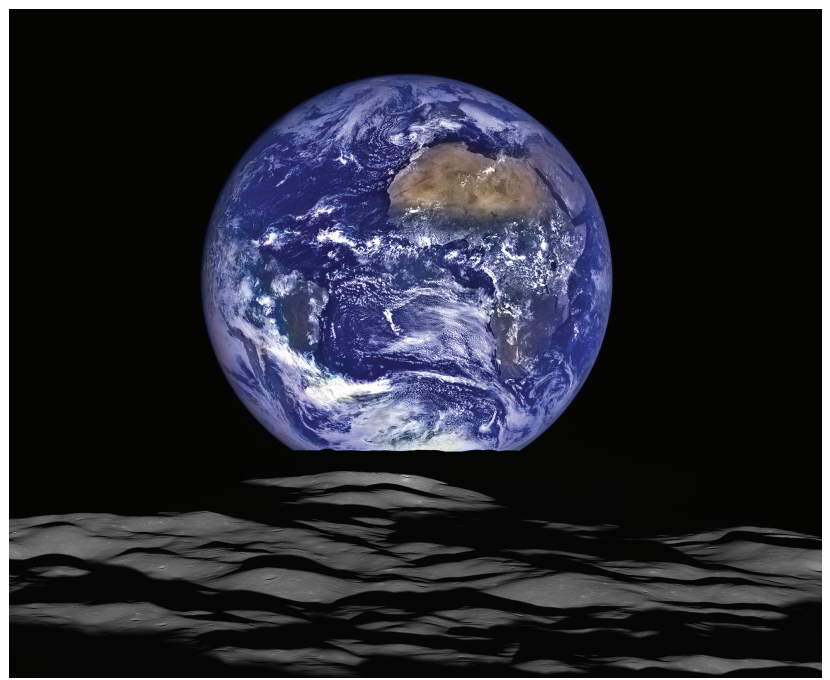

Figure 3. LRO Image of Earth [16]

LRO has experienced numerous anomalies with both of its star trackers. Some of the resets have been the result of radiation events. For most of the anomalous events the trackers issue a 'State 1553 Data Fault Detection', which seems to be due to an ASIC RAM overflow after an expected occultation. It appears that too much bright light remains in the FOV for a period of time after an occultation should have ended. Software has been uploaded to the star trackers preventing them from staying in an initialization mode too long following the RAM overflow events. The thermal electric cooler is disabled in the initialization mode. The updates allow for an autonomous promotion to standby mode, preventing increased CCD temperatures. Eventually the star trackers return to an attitude determination mode once the bright light clears from the FOV. The LRO team is continuing the investigation into the anomalous events, hoping to better determine what causes the extended period of bright light after a predicted occultation.

Actuators-None of the current SSMO missions have experienced a complete reaction wheel failure. Reaction wheel 3 on LRO stopped for a period of time in 2011. The actual cause could not be determined. An anomaly review team analyzed both a mechanical failure and an electrical failure, 


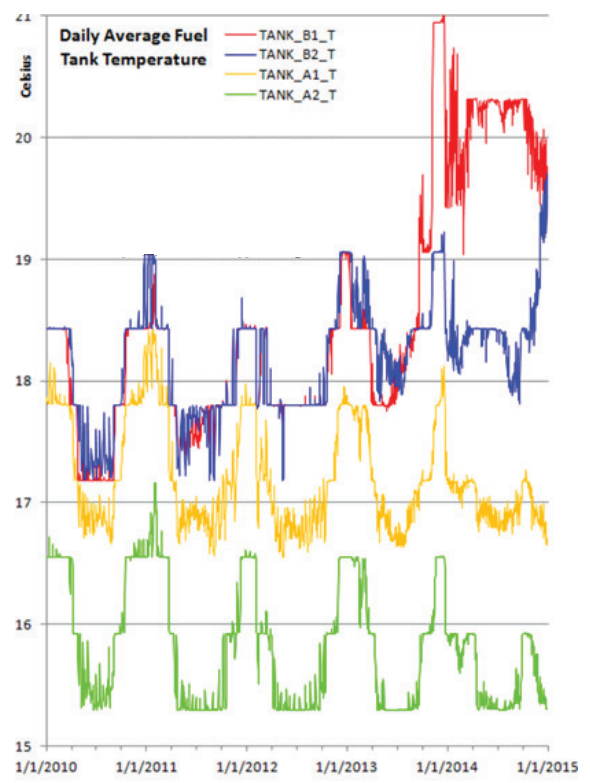

Figure 4. ACE Propulsion Tank Temperatures

but the telemetry was inconclusive to determine precisely the cause of the failure. The wheel was successfully restarted and the control system performance was monitored. Flight software updates have been made to detect a wheel failure and transition the control system to three-wheel operations. Reaction wheel 3 has been performing nominally ever since the anomaly. SSMO missions now routinely trend reaction wheel performance and monitor the reaction wheel drag.

ACE, launched in 1997, provides near-real-time solar wind information over short time periods. When reporting space weather, ACE can provide an advance warning (about one hour) of geomagnetic storms that can overload power grids, disrupt communications on Earth, and present a hazard to astronauts. ACE spins at approximately $5 \mathrm{rpm}$ and has four blow-down fuel tanks split into two pairs, A and B. In 2013 the B tanks reported increased temperatures and by 2015 maneuvers began to underperform by as much as $50 \%$. The cause was determined to be from pressurant escaping into the fuel lines as the two B side tanks emptied. Figure 4 shows the tank temperatures over a five year period. The fuel lines of the four tanks are interconnected but the pressurant lines are not. This allows the one pair of tanks to have more fuel mass than the other. A detailed study was conducted of ACE thruster performance and fuel distribution ([5]), which led to the decision to close the B-side thruster latch valves in March 2015. Since that time the ACE maneuvers have performed nominally. ACE is predicted to have another 10-11 years of fuel remaining.

\section{Power}

AIM was launched in 2007 with the primary goal of helping scientists understand whether the clouds' ephemeral nature, and their variation over time are related to Earth's changing climate; and to investigate why they form in the first place. Early in the mission the spacecraft receiver became unable to achieve subcarrier lock on command signals, forcing the project to develop a method to send commands via Morse code. Due to the greatly diminished commanding capability, improved autonomy was developed to allow the spacecraft and instruments to continue to operate in the new condition. [6] Over time the orbit has precessed, resulting in different beta angle conditions on the spacecraft. With the fixed solar array and the current operational modes, power concerns developed. In late July 2016 an incorrect determination of the eclipse periods by one of the science instruments forced a transition to a contingency mode where instruments are powered off. In September, the spacecraft began the transition back to science mode by entering an intermediate mode developed as part of the Morse code operations. In the intermediate mode the spacecraft is nadir pointing. The duration of time spent in this pointing profile coupled with a higher beta angle and a degraded battery did not provide sufficient conditions to remain power positive. An undervoltage threshold tripped, turning off heaters, the Z-axis reaction wheel, and the star tracker; in addition to transitioning the spacecraft back into the contingency mode. Reduced loads and a Sun pointing mode caused the battery pressure to increase. AIM uses a pressure-based state of charge calculation to drive the battery charge controller. The increase in the pressure resulted in an increase in the calculation of the state of charge, which lead to the charge controller placing the battery in a constant trickle charge even though the battery voltage indicated that it was being under charged. A carefully orchestrated increase in the spacecraft load profile was made to drive the pressure down while maintaining adequate battery voltage, which returned the battery to near-nominal activity. The exception being that the SOC was now slightly higher than before, but only by an amount consistent with the battery's degradation. In order to return to a nominal charging profile, variables used in the on board calculation of the state of charge were inflated proportionally to the observed degradation of the battery. With the battery back in the nominal state, changes were made to reduce the time spent in the transitional mode; thereby reducing the probability of reoccurrence. AIM expects to be back in science operations in late October 2016.

Swift was launched in 2004. Swift was designed to solve the mystery of the origin of gamma-ray bursts, which scientists believe are the birth cries of black holes. By late 2015, Swift had spotted 1000 Gamma Ray Bursts. [18] Over the mission lifetime, the potentiometers that measure the solar array gimbal angle have become noisier. In 2015, Swift entered a safe mode due to prolonged noise from both potentiometers on the plus Y solar array. The solar array gimbal controller compares the potentiometers to each other and compares each to the estimated gimbal angle from a step counter. The average of the potentiometers is also compared to the estimated angle. When one potentiometer exceeds the estimated angle in magnitude by more than 10 degrees it is discarded (meaning the new average is just from a single potentiometer). When the magnitude of the average of the potentiometers exceeds the estimated angle by 10 degrees for a set period of time, the spacecraft transitions to a safe mode. In order to avoid increased transitions to safe mode due to the increased noise, the threshold for entering safe mode was raised to 15 degrees. In September 2016 the potentiometers exceeded the 15 degree error trip and a command was issued to enter safe mode. However, the solar array gimbal was in the process of rotating when the command was issued to transition to safe. The Swift solar array gimbals do not have the ability to reverse direction in the middle of a slew. The commanded gimbal angle was changed, but the gimbal continued to rotate in the current (wrong) direction the prescribed number of steps to get to what should have been the new gimbal angle. The control system uses the coarse sun sensor data to point the $\mathrm{X}$-axis towards the sun, keeping 
the instrument boresight 90 degrees from the sun. However, since the estimated gimbal angle was incorrect, in actuality the spacecraft attempted to point the Z-axis towards the sun, which caused the instrument shutter to close to protect the instrument. A proposed fix to this scenario is still under investigation.

As mentioned above, it is believed that MMS4 was struck by a micrometeoroid in February 2016. The MMS spacecraft have five shunts comprised of four $180 \mathrm{ohm}$ resistors in parallel. They route excess electrical power generated by the solar arrays. The excess power is dissipated to space through four radiator panels. The micrometeoroid hit took out one of the four resistors on Shunt 2. This caused the overall resistance to increase from 45 to 60 ohms with a $25 \%$ loss of shunting capability from Shunt 2, equating to a $5 \%$ loss of shunting capability for the entire spacecraft. The power system electronics control design can tolerate the loss of an entire shunt and still achieve linear regulation in its control loop. The strike was picked up by the accelerometer and one of the science instruments, the nutation angle and spin axis underwent small changes, and the star tracker had spikes in the number of bright, non-star objects in the field of view. The boresight of the star tracker is nearly aligned with the normal direction to the radiator panels. The star tracker rebooted after not being able to produce a valid attitude solution for a prolonged period and returned to normal operation. [8]

\section{Mission OpERATIONS IMPROVEMENTS}

SSMO monitors the performance and operations of all the missions in its portfolio. There are numerous activities underway to improve operations both on the ground and inflight. This section highlights a few of the current activities currently underway in SSMO.

\section{$L R O$}

After the gyro anomalies on STEREO, an effort began to develop a gyroless operating mode for LRO. The flight software can currently compute the angular velocity by differentiating the star tracker quaternions for use in a safe operating mode, but this angular velocity estimate does not meet the science pointing requirements. Testing has begun on a complimentary filter method that combines the star tracker differentiated quaternions with a rate estimate based on the PID controller feedback torque. The combined rate estimate would replace the gyro estimate in the onboard Kalman filter. [9] Alternatively, the Kalman filter could be updated to estimate the spacecraft rate in the state, rather than estimating a gyro bias in the state. [10] This approach would require significantly more changes to the current flight software than the complimentary filter, but is an option should the complimentary filter fail to meet the science pointing requirements. If a gyroless approach is successful for LRO, it will then be applied and tested on SDO.

LRO performs more than 20 thruster maneuvers per year to dump excess momentum in the reaction wheels. The maneuvers disrupt the science operations; three instruments have to be safed, and one requires 24 hours of decontamination following a thruster maneuver. An analysis effort has begun to optimize the momentum unloading for LRO. A tool was developed that can predict momentum buildup as a function of the beta angle. A linear regression analysis was performed on a full beta cycle spanning October 2014 through October 2015. The model developed was used to predict angular momentum buildup from October 2013 through October 2014

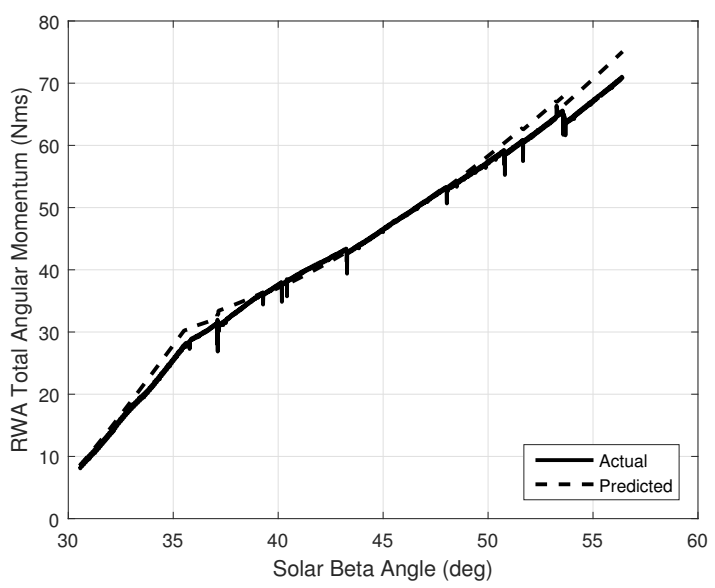

Figure 5. LRO Predicted and Actual Angular Momentum, September 1-27, 2016

and has since been used to inform the planning of momentum unloads in 2016. The predictions agree well with telemetry, as depicted for September 2016 in Figure 5. The higher accuracy angular momentum predictions provided by this tool allow for less frequent momentum unloads. The tool also provides insight as to when the momentum targets can be changed to allow an even longer period between momentum unloads. In addition, the tool quantifies the change in system angular momentum induced by slews frequently performed as part of nominal operations. The operations concept is currently being analyzed to determine when different momentum targets and particular slews can be used to lengthen the time between momentum unloads and save fuel. [17]

A Slew Verification Tool (SVT) was also developed for LRO to provide power and thermal predictions for the frequent slew maneuvers. The tool advises the operations team if it is safe to perform a particular slew. If the slew is questionable it provides a warning for the flight operations team to follow up with the power and thermal engineers for a more detailed analysis. The SVT thermal checks rely on calculations of the spacecraft geometry relative to the sun and moon in order to conservatively determine orientations that may be problematic for the instruments. If the model predicts that the instrument deck will be heated too much by either solar irradiance or the lunar surface, it will notify the operations team to investigate further. The SVT power checks take into account characteristics of the battery and the solar array in order to calculate the battery voltage and current. If the voltage drops below pre-defined limits, SVT will notify the operations team. The power calculation incorporates a loadbased safety factor in order to provide a conservative estimate of the battery voltage. Figure 6 shows battery telemetry in red and calculated battery voltage in white.

\section{$S D O$}

SDO is the first mission to be launched for NASA's Living With a Star (LWS) Program, a program designed to understand the causes of solar variability and its impacts on earth. SDO is contributing to the understanding of the sun's influence on earth and near-earth space by studying the solar atmosphere on small scales of space and time and in many wavelengths simultaneously. SDO is a sunpointing semi-autonomous spacecraft in a geosynchronous orbit that allows nearly continuous observations of the sun 


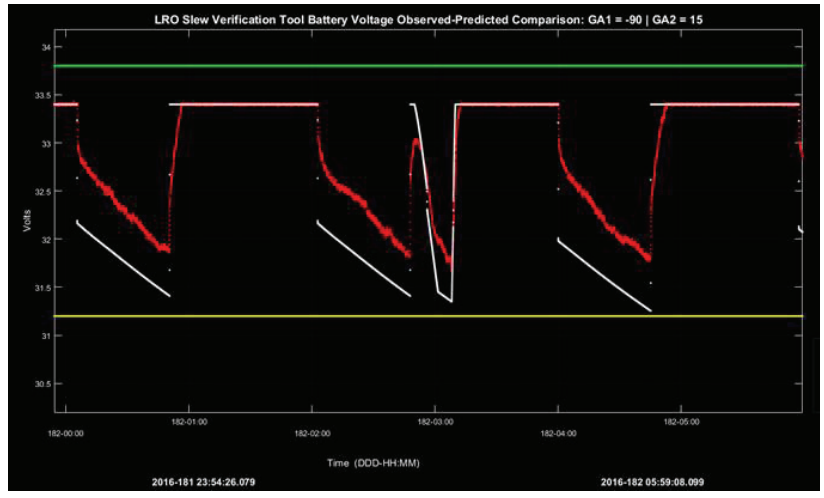

Figure 6. LRO Slew Verification Tool Battery Voltages (red $=$ telemetry, white $=$ estimated, yellow $=$ limit $)$

with a continuous science data downlink rate of 130 Megabits per second (Mbps). [11] The attitude control system must accurately point SDO at the sun and keep the roll about the sun correctly positioned with respect to the solar north pole. SDO is equipped with coarse sun sensors, a digital sun sensor, two star trackers, and three two-axis inertial reference units. In the fine-pointing mode, an instrument guide telescope is used to provide the $2-\operatorname{arcsec}(3 \sigma)$ accuracy. [12] The IRUs are temperature sensitive and were designed to operate in a stable thermal environment. However, the operation of the heaters produces a large amplitude, highfrequency transient in the battery. Early in the mission the decision was made to operate the IRUs without the heaters in order to preserve battery life. In late 2010, the current began to increase on IRU1, and in 2013 a decision was made to shut off IRU1. In 2015 the current started to increase on IRU2. The IRUs were intended to operate at approximately 70 degrees $\mathrm{C}$, but were instead operated between 26 and 32 degrees. Late in 2015 a test was conducted to evaluate the impact of turning on the IRU heaters over a two week period. The noise in the IRU measurements dropped and the estimated biases stabilized. The IRU currents also dropped. [13] Simultaneously, the Global Precipitation Mission was conducting a ground test of their battery (same battery as SDO) to determine the effects of micro-cycling the battery. The results indicated that after a year of running the highfrequency load the battery degradation was negligible. [14] In September 2016 a decision was made to turn on the SDO IRU heaters. As with the test in late 2015, the IRU currents dropped, the measurement noise decreased, and the biases stabilized. Figure 7 shows the transition in biases in the $\mathrm{X}$ and $\mathrm{Y}$ axes. The SDO instruments also noticed an improved response due to the improved pointing accuracy. Figure 8 shows one of the instrument stabilization responses when the IRU heaters were turned on. The battery performance will continue to be monitored on SDO.

\section{Fermi}

Fermi has a 2 -arcsec $(1 \sigma)$ pointing knowledge requirement, a 2-degree $(1 \sigma)$ pointing control requirement, and a requirement to slew 75 degrees in less than 10 minutes. Fermi is equipped with 3 star trackers (one is a cold spare), a block redundant IMU, four reaction wheels, coarse sun sensors, magnetometers, thrusters, magnetic torque rods, and a GPS receiver. The reaction wheels have been trended throughout the mission life, however sufficient statistics were not available to determine current performance as compared to the life of the mission. A tool was developed for the mission operations team that now produces the drag torque estimates

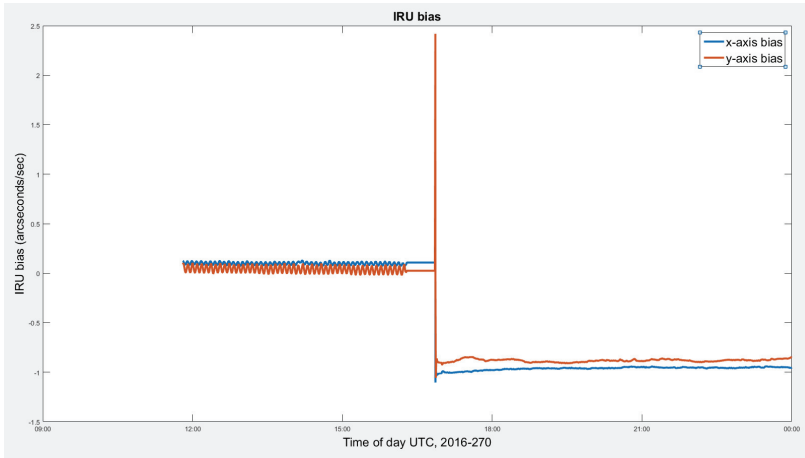

Figure 7. X and Y Axes Gyro Biases Following Heater Turn On

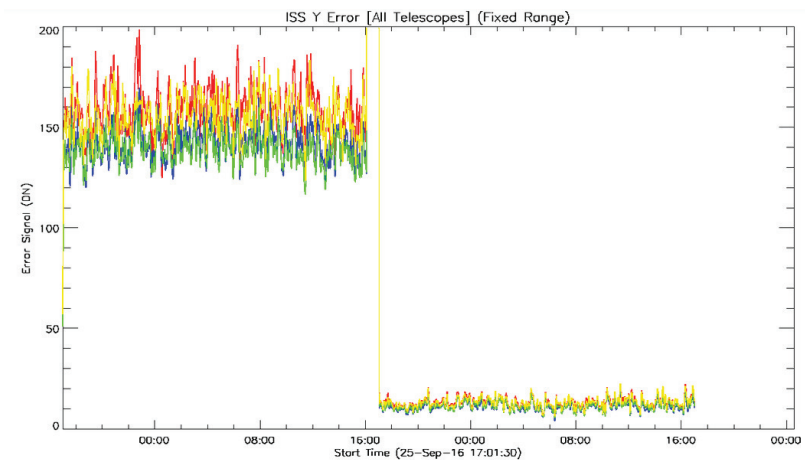

Figure 8. Instrument Stabilization System Following Heater Turn On

over both the current week and the life of the mission over the operating wheel speeds. In addition the drag torques over the life of the mission are plotted at wheel speeds ranging from $\pm 250 \mathrm{rpm}$ to $\pm 1000 \mathrm{rpm}$. Figures 9 and 10 show the evolution of the drag torque over the life of the mission at \pm $250 \mathrm{rpm}$.

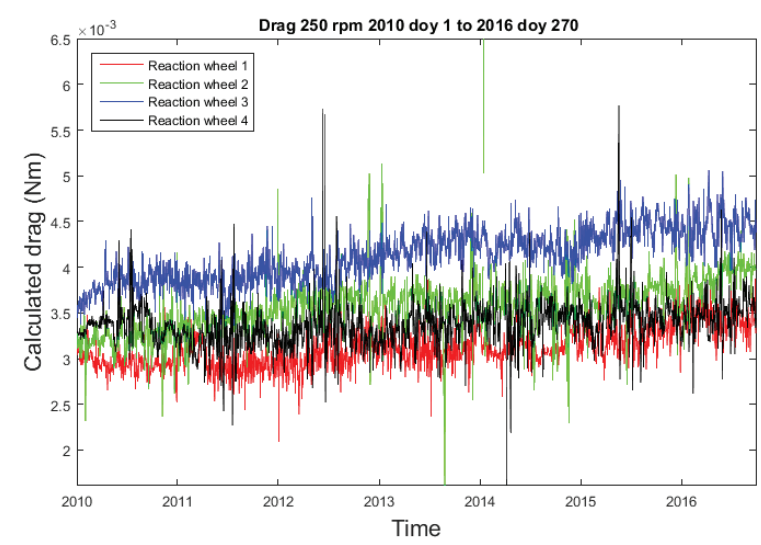

Figure 9. Fermi Reaction Wheel Drag Estimates at 250 rpm

\section{Data Delivery}

SSMO developed a multi-spacecraft data archive, access, trending and analysis tool that supports a large variety of NASA missions. It provides a single point system as a means for engineers to easily access and view mission data. Users 


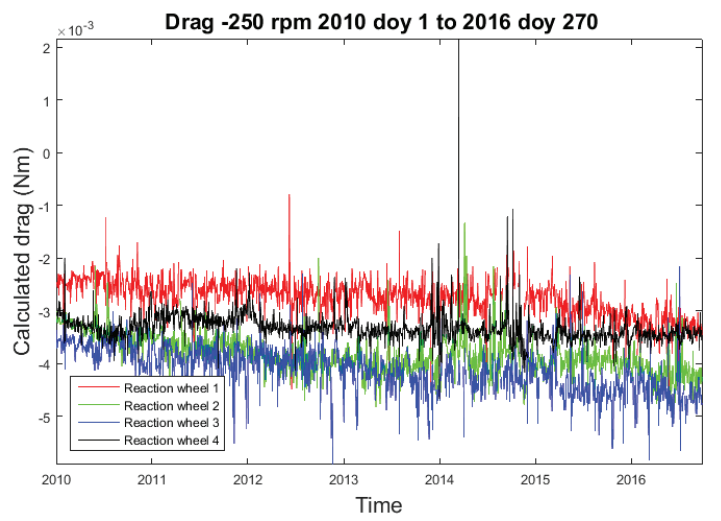

Figure 10. Fermi Reaction Wheel Drag Estimates at -250 rpm

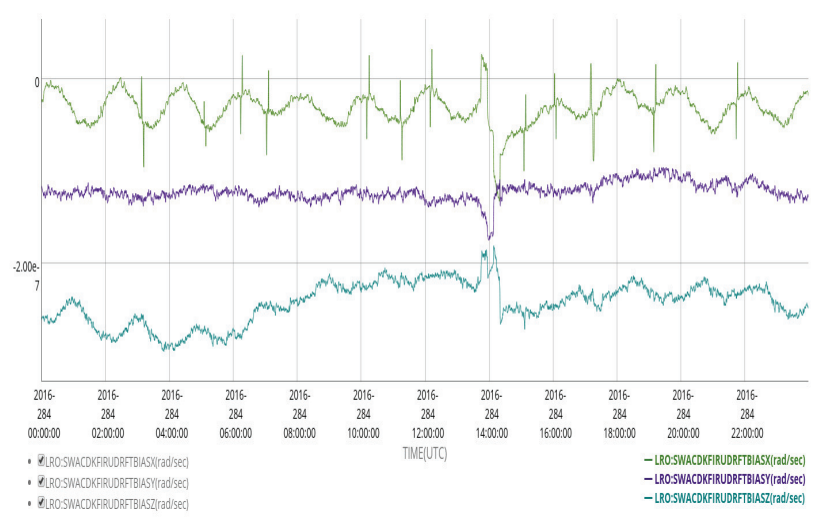

Figure 11. LRO Gyro Biases Plotted via SSMO Telemetry as a Service

can generate trending products, plots, reports, and export data to tools such as Excel and Matlab. However, as the number of missions and volume of data grew, the platform for hosting the data became too limited and the user interface cumbersome. In 2015 a new system, known as Telemetry as a Service, was developed. The service leverages open source software, standards, specifications, and architectures, utilizes modern web browsers, virtualizations and cloud services. The telemetry is now hosted on Amazon Web Services Gov Cloud and the user interface has been streamlined to easily search for mnemonics, even across multiple missions. [19] Figure 11 is an example plot showing the LRO onboard gyro biases over a day in late 2016. The system also allows the user to compare data across missions. It will serve as a valuable resource in data evaluation and trending.

\section{SUMMARY}

The NASA GSFC Space Science Mission Operations manages and operates a diverse fleet of spacecraft. The spacecraft range in age from the recent launch of OSIRIS-REx in September 2016 to over 20 years for SOHO and Wind. The spacecraft vary in size, orbital regime, control mode, number of instruments, and ground system complexity. This paper highlighted a number of the systems engineering challenges and operational improvements that are underway in SSMO.
The spacecraft continue to provide exciting scientific observations about the Sun, Moon, Mars, and soon an asteroid when OSIRIS-REx reaches Bennu.

\section{APPENDICES}

\section{A. ACRONYMS}

ACE
ACS
AIM
ARTEMIS

Advanced Composition Explorer Attitude Control System

Aeronomy of Ice in the Mesosphere Acceleration, Reconnection, and Turbulence and Electrodynamics of the Moon's Interaction with the Sun

ASC
CCD
IBEX
IRIS
Autonomous Star Tracker Charge Coupled Device Interstellar Boundary Explorer Interface Region Imaging Spectrograph

$\begin{array}{ll}\text { IRU } & \text { Inertial Reference Unit } \\ \text { FOV } & \text { Field of View } \\ \text { FPGA } & \text { Field-Programmable Gate Array } \\ \text { GEO } & \text { Geosynchronous Orbit } \\ \text { GSFC } & \text { Goddard Space Flight Center } \\ \text { HEO } & \text { Highly Elliptic Orbit } \\ \text { LEO } & \text { Low Earth Orbit } \\ \text { L1 } & \text { Earth/Sun Libration Point 1 } \\ \text { LRO } & \text { Lunar Reconnaissance Orbiter } \\ \text { MAVEN } & \text { Mars Atmosphere and Volatile Evo- } \\ & \text { lution } \\ \text { MMS } & \text { Magnetospheric Multiscale } \\ \text { OSIRIS-REx } & \text { Origins, Spectral Interpretation, } \\ & \text { Resource Identification, Security, } \\ & \text { Regolith Explorer } \\ \text { RAM } & \text { Random Access Memory } \\ \text { RHESSI } & \text { Reuven Ramaty High Energy Solar } \\ & \text { Spectroscopic Imager } \\ \text { RPM } & \text { Revolutions Per Minute } \\ \text { SDO } & \text { Solar Dynamics Observatory } \\ \text { SOHO } & \text { Solar and Heliophysics Observa- } \\ & \text { tory } \\ \text { SSMO } & \text { Space Science Mission Operations } \\ \text { STEREO } & \text { Solar Terrestrial Relations Observa- } \\ & \text { tory Verification Tool } \\ \text { SVT } & \text { Slew Verifist } \\ \text { THEMIS } & \text { Time History of Event and } \\ & \text { Macroscale Interactions during } \\ \text { TIMED } & \text { Substorms } \\ & \text { Thermosphere Ionosphere Meso- } \\ \text { sphere Energetics and Dynamics }\end{array}$

\section{ACKNOWLEDGMENTS}

The authors wish to thank Russell DeHart, Brian Lounsbury, and Devin Poland of the SSMO Systems Engineering Team for their input and insight into the preparation of this paper, and their excellent support of the many missions within SSMO.

\section{REFERENCES}

[1] 2015 Goddard Space Flight Center Annual Report. site: http://www.nasa.gov/centers/ goddard/about/rep_plan.html.

[2] Leonard David, Saving SOHO, Aerospace America, May 
1999, pp. 60-67.

[3] Magnetic Mutliscale Web site: https: / / www. nasa. gov/mission_pages/mms/index.html.

[4] M. Farahmand, A. Long, and R. Carpenter. Magnetospheric Multiscale Mission Navigation Performance Using the Goddard Enhanced Onboard Navigation System. In Proceedings of the 25th International Symposium on Space Flight Dynamics, www.issfd.org, 2015.

[5] R. DeHart, E. Smith, and J. Lakin. Fuel Distribution Estimate Via Spin Period to Precession Period Ratio for the Advanced Composition Explorer. In Proceedings of the 25th International Symposium on Space Flight Dynamics, www.issfd.org, 2015.

[6] Deb McCabe, Sean Ryan, Dave Welch, and John Fulmer. AIM Autonomy Development - Long Term Care for a Deaf Spacecraft. SpaceOps 2008 Conference, Heidelberg, Germany

[7] Marshall Smart, Ratnakumar Bugga, Larry Whitcanack, Frank Puglia, Stuart Santee, and Robert Gitzendanner. Performance Testing of Yardney Li-Ion Cells and Batteries in Support of Future NASA Missions. 7th International Energy Conversion Engineering Conference, Denver, Colorado, August 2009.

[8] Trevor Williams, Seth Shulman, Joseph Sedlak, Neil Ottenstein, and Brian Lounsbury. Magneticospheric Multiscale Mission Attitude Dynamics: Observations from Flight Data, AIAA SPACE 2016, Long Beach, California.

[9] Phil Calhoun. MIMU Failure Risk Mitigation Trade Study, NASA GSFC internal presentation, August, 2014.

[10] Julie K. Thienel and F. Landis Markley. Comparison of Angular Velocity Estimation Methods for Spinning Spacecraft, AIAA Guidance, Navigation, and Control Conference, Portland, Oregon, 2011.

[11] Solar Dynamics Observatory site: http://sdo. gsfc.nasa.gov/.

[12] James R. O’Donnell Jr., Kristin L. Bourkland, Oscar C. Hsu, Kuo-Chia (Alice) Liu, Paul A. C. Mason, Wendy M. Morgenstern, Angela M. Russo, Scott R. Starin, Melissa F. Vess. Solar Dynamics Observatory Launch and Commissioning, AIAA/AAS Astrodynamics Specialist Conference, Girdwood, Alaska, August, 2011.

[13] Devin Poland. SDO Heater Turn On Analysis, Internal GSFC Memo, September, 2016.

[14] Paper details to be supplied by November 2016.

[15] Fermi Gamma Ray Telescope website: https://www.nasa.gov/content/ fermi-gamma-ray-space-telescope.

[16] Lunar Reconnaissance Orbiter: https: //www.nasa.gov/image-feature/goddard/ lro-earthrise-2015.

[17] Russell DeHart. Multi-Week Predictions of Lunar Reconnaissance Orbiter Reaction Wheel Assembly Angular Momenutm Using Beta Angle Regression Models and Analysis of Previous Reaction Wheel Assembly Responses to Spacecraft Activities, To Be Included in AIAA SciTech 2017, Grapevine, Texas, January, 2017

[18] Swift website: https://www.nasa.gov/ mission_pages/swift/main.

[19] Haisam Ido, SSMO Data Access Tool, internal GSFC presentation, January 2015.

\section{BIOGRAPHY}

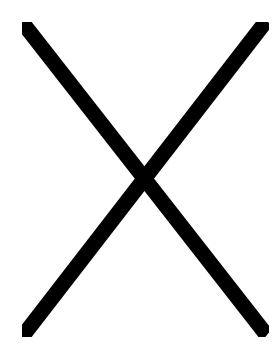

Julie Thienel received her B.S. in Aerospace Engineering from the University of Arizona in 1986, M.S in Applied Physics from the Johns Hopkins University in 1989, and a Ph.D. in Aerospace Engineering from the University of Maryland in 2004. She is currently the lead Systems Engineer for the NASA GSFC Space Science Mission Operations.

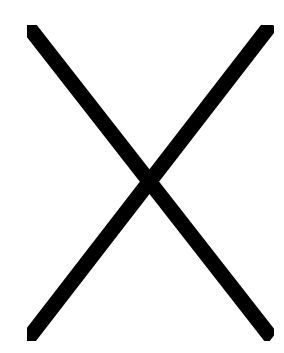

Rick Harman received his B.S. degree in Physics in 1986 from Virginia Tech and M.S. in Electrical Engineering from the Johns Hopkins University in 1991. He is currently the Deputy Project Manager for the NASA GSFC Space Science Mission Operations. 